\title{
Protein-glycan interactions in the control of innate and adaptive immune responses
}

\begin{abstract}
Yvette van Kooyk ${ }^{1}$ \& Gabriel A Rabinovich ${ }^{2}$
The importance of protein glycosylation in the migration of immune cells throughout the body has been extensively appreciated. However, our awareness of the impact of glycosylation on the regulation of innate and adaptive immune responses is relatively new. An increasing number of studies reveal the relevance of glycosylation to pathogen recognition, to the modulation of the innate immune system and to the control of immune cell homeostasis and inflammation. Similarly important is the effect of glycan-containing 'information' in the development of autoimmune diseases and cancer. In this review, we provide an overview of these new directions and their impact in the field of glycoimmunology.
\end{abstract}

Glycans cover the surfaces of all mammalian cells: they are added to protein and lipid backbones-a process called glycosylation-during the biosynthesis as these molecules are transported from the endoplasmic reticulum to the Golgi and the cell membrane. Glycosylation is mediated by a coordinated set of 'machinery' that involves glycosyltransferases, enzymes that catalyze the transfer of a sugar from a nucleotide sugar donor or a lipid sugar to a substrate, and glycosidases, enzymes that catalyze the hydrolysis of glycosidic bonds in glycan structures. Genes encoding this glycosylation machinery represent $>1 \%$ of the total genome; to date, more than 100 glycosyltransferases and glycosidase enzyme genes have been cloned that contribute to the selective addition and/or removal of particular monosaccharides to or from a 'growing' glycan structure ${ }^{1}$. The expression of these enzymes is highly regulated according to cell metabolism, activation, microenvironmental changes and aging. Therefore, glycosylation itself can give different structural variations to a given protein, leading to identical proteins linked to different glycan structures (glycoforms). This diversity of glycans and enzymes gives the mammalian glycome an estimated variation of thousands of potential glycan structures, which adds to the diversity already created by the proteome. Within the mammalian glycosylation repertoire, $\mathrm{N}$-linked glycosylation, $\mathrm{O}$ linked glycosylation and glycosaminoglycans have been identified (Fig. 1). The presence of potential $\mathrm{N}$ - and O-linked glycosylation sites in a protein backbone, together with the presence or absence of glycosyltransferases and glycosidases in the endoplasmic reticulum, are key elements in determining the extent and nature of the glycosylation of a given protein.

${ }^{1}$ Department of Molecular Cell Biology and Immunology, VU University Medical Centre, van de Boechorststraat 7, 1081BT Amsterdam, The Netherlands.

2Laboratory of Immunopathology, Institute of Biology and Experimental

Medicine, National Council of Scientific and Technological Research,

C1428ADN, Ciudad de Buenos Aires, Argentina.

Correspondence should be addressed to Y.v.K. (y.vankooyk@vumc.nl) or G.A.R (gabyrabi@ciudad.com.ar).

Published online 19 May 2008; doi:10.1038/ni.f.203
Many eukaryotic and prokaryotic pathogens express glycoconjugates on their surface or on secreted products. Glycans synthesized by prokaryotic and eukaryotic pathogens (for example, meningococci, Trypanosoma and Helicobacter) can include terminal glycan structures that are similar to those found in mammalian glycans (so-called 'molecular mimicry'). However, some pathogens, including Schistosoma mansoni and Mycobacterium tuberculosis, may also express specific glycan epitopes ${ }^{2}$.

\section{Glycan recognition by proteins}

Within the immune system, various classes of glycan-binding receptors (lectins) exist that recognize specific glycan structures presented on a protein backbone or lipid structure. These receptors are secreted or are found on the cell surface of immune cells. In this review, we will focus on three receptor families that are involved in glycan recognition: galectins, C-type lectins and siglecs ${ }^{3-6}$. The glycan specificity of these receptors is well documented, as is their cell-specific expression and secretion (Supplementary Table 1 online). Whereas galectins are secreted, most C-type lectins and all known siglecs are membrane-bound proteins. All these lectins contain one or more carbohydrate-recognition domains (CRDs) responsible for glycan binding.

Galectins are a family of soluble lectins defined by a common structural fold and a conserved CRD of about 130 amino acids that recognizes glycans containing the disaccharide $N$-acetyllactosamine (Gal- $\beta(1-4)$ GlcNAc: Gal, galactose; GlcNAc, $N$-acetylglucosamine) $)^{3,7}$. As many as 15 galectins have been identified in mammals ${ }^{8}$. Some galectins (galectins $1,2,5,7,10,11,13,14$ and 15), which are traditionally classified as 'prototype' galectins, have one CRD that can dimerize, whereas others (galectins 4, 6, 8, 9 and 12), so called 'tandem-repeat galectins', contain two homologous CRDs in tandem in a single polypeptide chain, separated by a linker of up to 70 amino acids. Galectin-3 is, as far as we know, unique in that it contains a CRD connected to a non-lectin $\mathrm{N}$-terminal region (about 120 amino acids) that is responsible for oligomerization of the lectin and ligand cross-linking ${ }^{3,9,10}$. The presence of more than one oligosaccharide-recognizing CRD in a galectin-1 homodimer makes the homodimer well suited for mediating cell adhesion, eliciting signaling 


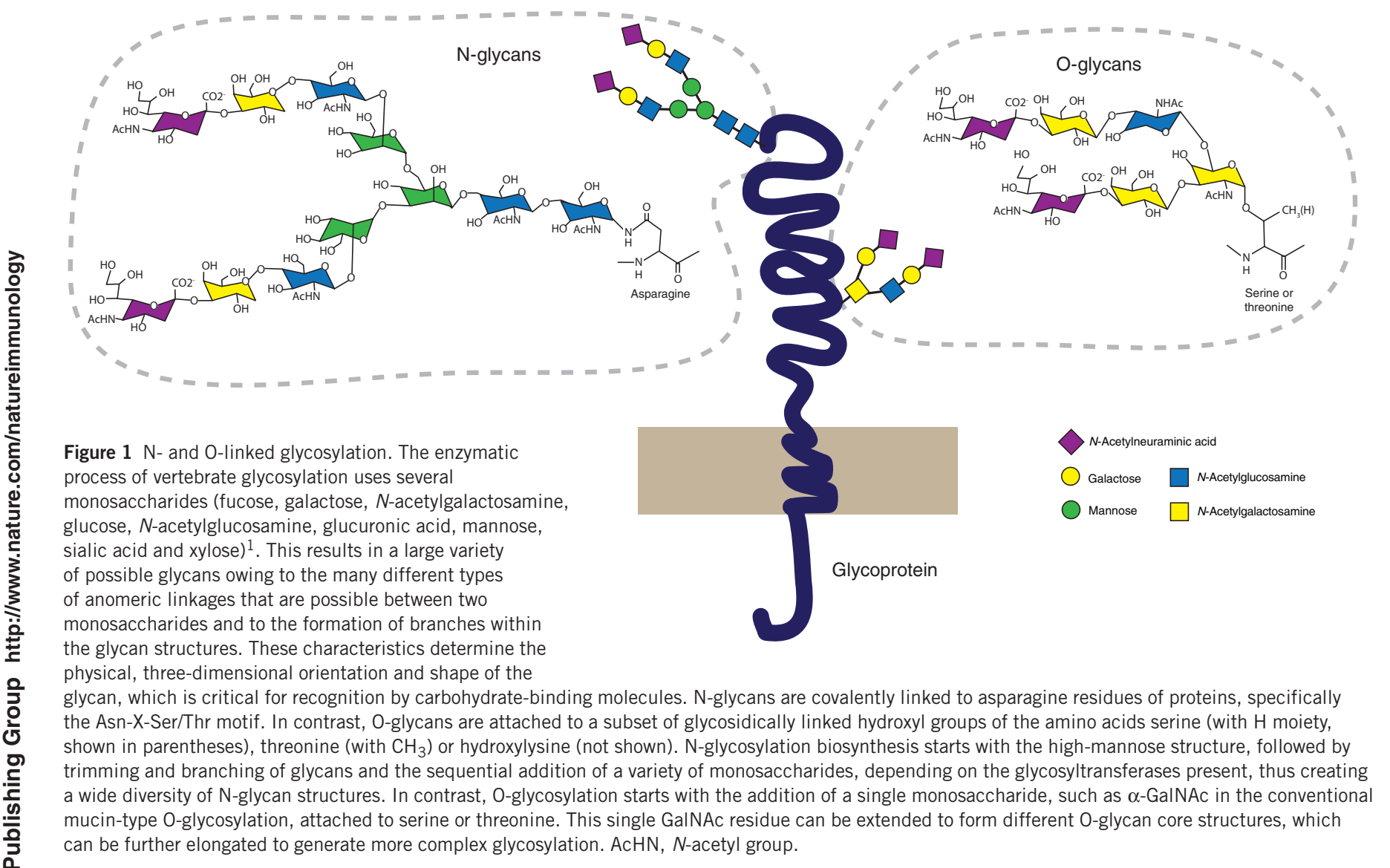

and forming cell-surface lattices ${ }^{8,9}$, whereas tandem-repeat galectins are intrinsically bivalent, although the two CRDs may recognize different saccharide ligands ${ }^{3}$. Some galectins, such as galectin-1 and galectin-3, are distributed in a wide variety of tissues, whereas others, such as galectin-4 and galectin-10, have more restricted localizations. In the immune system, galectins are mostly expressed by activated but not resting $\mathrm{T}$ and $\mathrm{B}$ cells, and they are significantly upregulated in activated macrophages and regulatory $\mathrm{T}$ cells ${ }^{8,9}$. Although these soluble lectins do not have the signal sequence required for the classical secretion pathway, they are secreted into the extracellular milieu through an atypical secretory mechanism ${ }^{3,8,9}$.

C-type lectin receptors (CLRs) are calcium-dependent carbohydratebinding proteins and consist of a large family of receptors (60-80) that is further divided into subfamilies ${ }^{6,11,12}$ (http://www.imperial.ac.uk/ research/animallectins/ctld/mammals/humandata.html). Several of these C-type lectins (the natural killer (NK) cell receptor-like family) have no classical CRD domains and thus are not involved in protein-glycan interactions ${ }^{5,6,11}$. However, most CLRs are glycan-binding receptors, as they carry one or more CRD regions. C-type lectins can be divided into two categories on the basis of an amino acid motif involved in glycan recognition and coordination of the $\mathrm{Ca}^{2+}$ ion. The mannose-specific C-type lectins contain an EPN (Glu-Pro-Asn) amino acid motif and have a specificity for mannose- and/or fucose-terminated glycans. In contrast, the galactose-specific C-type lectins contain the QPD (GlnPro-Asp) sequence in the CRD and recognize galactose-terminated or $\mathrm{N}$-acetylgalactosamine (GalNAc)-terminated glycan structures ${ }^{5,6,12}$. The type II subfamily of CLR, of which 17 members have been cloned in human, is mainly restricted to antigen presenting cells (APCs) such as macrophages and dendritic cells (DCs); some have also been iden- tified on NK cells or endothelial cells ${ }^{5,6,12}$. Maturation of DCs often results in loss of CLR expression ${ }^{12}$. CLRs such as DC-SIGN, L-SIGN, mannose receptor, macrophage galactose-specific lectin (MGL) and langerin are well characterized for their specificity for high-mannose, fucose-containing glycans (Lewis ${ }^{\mathrm{a}, b, \mathrm{x}, \mathrm{y}}$ ), GalNAc or GlcNAc ${ }^{5,13-16}$. These glycan structures can be expressed by mammalian cells or by pathogens, reflecting their dual function in host-pathogen recognition and immune cell responses. In contrast, dectin-1, a $\mathrm{Ca}^{2+}$-independent CLR, is specific for $\beta$-glucan, a fungus-specific glycan ${ }^{17,18}$. Typically, CLRs show specificity for clustered glycans (polyvalency) that increases their affinity ${ }^{13,19}$. Polyvalency is often achieved by the expression of glycans on protein backbones that contain multiple repeating subunits, such as mucin-like molecules, or by the expression of multiple copies of a given protein carrying the specific glycans. Such enhanced avidity of a given CLR coincides with the formation of multimers of these receptors in cell membranes ${ }^{6,15}$. Finally, C-type lectins have been described to function as patterns for pathogen recognition receptors and as receptors for antigen uptake, signaling and cell adhesion ${ }^{20}$.

Siglecs (sialic acid-binding immunoglobulin-like lectins) are a family of type I membrane proteins with variable numbers of immunoglobulin domains. On the basis of sequence similarity and evolutionary conservation, they can be divided into two types: CD33-related siglecs (Siglecs 3, $5,6,7,8,9,10,11$ and 14) and a group consisting of sialoadhesin (Siglec-1 or CD169), MAG (Siglec-4) and Siglec-2 (CD22) $)^{4}$. These glycan-binding proteins are well known for their specificity for sialic acid-containing glycans (of which $N$-acetylneuraminic acid is the most common) 4,21,22 $^{\text {. }}$ Sialylated structures are often found on the nonreducing ends of oligosaccharides chains of N- or O-linked glycans or on glycosphingolipids. All siglecs recognize sialylated structures but can differentially 
discriminate among specific linkages $(\alpha(2-3), \alpha(2-6)$ or $\alpha(2-8))$ by which the sialic acid structures are linked to the underlying glycan structure and can selectively distinguish structural features of the glycans themselves ${ }^{4}$. For the CD33-related siglecs and CD22 (Siglec-2), the presence of immunoreceptor tyrosine-based inhibitory motifs (ITIM) or ITIMlike motifs in the cytoplasmic tail can modulate cellular functions by recruitment of signaling molecules ${ }^{4}$. At present, 13 members of the siglec family, which are widely expressed on immune cells, including neutrophils, monocytes, B cells, DCs, NK cells, eosinophils and basophils, have been identified in humans. However, expression on human T cells is barely detectable. In addition, sialic acid structures can be found on pathogens, illustrating the bifunctional role of siglecs in both pathogen recognition and intercellular communication of immune cells ${ }^{4}$.

Uniquely (to our knowledge), siglecs can mediate cis and trans interactions with sialylated glycans ${ }^{4}$. In the case of cis interactions, the siglec is often masked by low-affinity ligands on neighboring membrane receptors. Because the local concentration of sialic acid on the surface of immune cells is very high, the siglec-binding sites may typically be masked by cis interactions with glycan ligands expressed on the same cell, indicating that cis interactions may play a dominant role in modulating immune responses. However, cis interactions do not necessarily prevent trans interactions ${ }^{23}$.

\section{Control of innate immune responses}

The capacity to recognize glycans from divergent organisms such as bacteria, yeast, viruses and helminth parasites underlies a mechanism for pathogen recognition. This is illustrated by Toll-like receptor (TLR) activation of innate immune system through binding to bacterial glycans ${ }^{24}$. Likewise, many CLRs are expressed on innate immune cells, particularly macrophages and DCs; these APCs are the first line of defense responsible for clearing pathogens, and they subsequently initiate adaptive immune responses. The DC-expressed C-type lectins mannose receptor (CD206), DC-SIGN (CD209), MGL (CD301) and dectin-1, as well as langerin (CD207) on Langerhans cells, are involved in glycan-mediated pathogen recognition and internalization of antigen for loading on major histocompatibility complex (MHC) class I and II molecules (Fig. $2)^{12,20,25}$. Thus, CLRs can function as pathogen recognition receptors that favor enhanced antigen presentation and antigen-specific $\mathrm{T}$ cell induction ${ }^{26}$. However, the glycans that CLRs recognize are often not actually pathogen specific; for example, many similar glycans can be found in mammalian cells. Yet some pathogen-specific glycans do occur, such as pseudo-Lewis ${ }^{\mathrm{y}}$, a pathogen-specific glycan structure recognized by DC-SIGN ${ }^{27}$.

Many reports have provided evidence that CLRs are signaling receptors that, together with activated TLRs, 'relay information' about specific pathogens into DC differentiation and immune-response programs by affecting the expression of cytokine genes ${ }^{5,16,18,28-30}$. A well-described example is the signaling function of the CLR dectin-1 upon recognition of fungal $\beta$-glucan structures, which triggers signaling by the Syk tyrosine kinase and activates the p38, Erk and JNK kinase cascades and the transcription factor NF- $\mathrm{BB}$ (ref. 18). For this signaling, the adaptor CARD9, which forms complexes with signaling molecules Bcl-10 and MALT1, is required, thus defining a pathway by which a CLR, independently of TLRs, regulates innate gene expression ${ }^{31}$. Recently, the CLR DC-SIGN has also been shown to signal upon recognition of pathogen glycan components, though in this case it does so through activation of the kinase Raf-1, which translates the signal into phosphorylation and subsequent acetylation of the activating NF- $\mathrm{KB}$ subunit p65 and thus increases transcription of specific cytokine genes ${ }^{28}$. Distinct from dectin signaling, DCSIGN-mediated signaling is dependent on TLR-induced activation of $\mathrm{NF}-\kappa \mathrm{B}$ and can in fact modulate TLR-dependent signaling ${ }^{28}$.

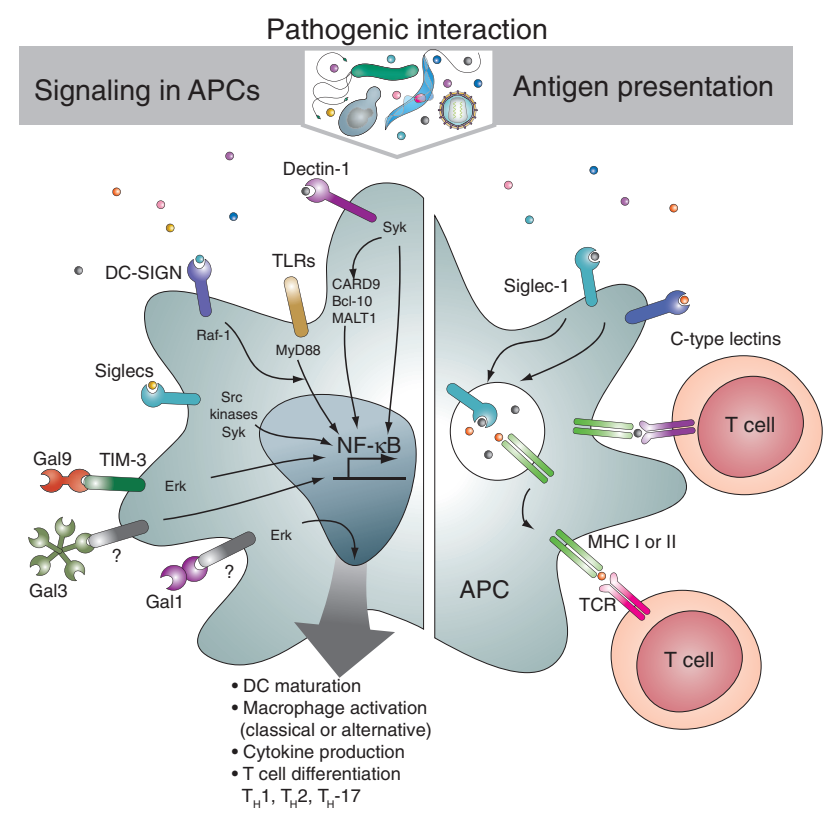

Figure 2 Glycan-binding proteins involved in pathogen interaction leading to antigen presentation and signaling in APC. Many C-type lectins and several siglecs (Siglecs 1, 5, 7 and $\mathrm{H}$ ) are pathogen recognition receptors that interact with specific glycan structures on these pathogens, such as Lewis ${ }^{x, y}$, GlcNAc, high-mannose structures, sialic acids and GalNAc, and mediate internalization and enhanced antigen processing and presentation by $\mathrm{MHC}$ class I and II. Soluble galectins can interact with pathogens, forming lattices and initiating innate immune responses. Pathogen recognition by C-type lectins and siglecs expressed on APCs can lead to various intracellular signaling processes and modulate TLR signaling or elicit signaling independently of TLR activation. Galectins can interact with certain glycoproteins expressed by APCs, thereby modulating APC maturation and cytokine production. Gal, galectin; MyD88, a common adaptor protein involved in TLR signaling.

Pathogens that simultaneously target CLRs and TLRs have been shown to modulate DC responses depending on their pathogen-specific glycan signatures, which, as described earlier, critically affect CLR binding. High-mannose structures on the cell-wall component ManLAM of M. tuberculosis 'target' the mannose receptor and DC-SIGN to induce signaling that leads to the production of anti-inflammatory cytokines such as interleukin (IL)-10 (refs. 20,26), whereas phase variants of Helicobacter pylori expressing the Lewis ${ }^{\mathrm{x}}(\mathrm{CD} 15)$ and Lewis ${ }^{\mathrm{y}}(\mathrm{CD} 174)$ glycan antigens on the lipopolysaccharide O-antigen 'target' DC-SIGN on DCs to induce signaling that leads T cells to differentiate into Thelper type $2\left(\mathrm{~T}_{\mathrm{H}} 2\right)$ cells $^{32}$. Similarly, $S$. mansoni soluble egg antigens expressing fucosylated GalNAc- $\beta(1-4)-G l c N A c$ sequences and Lewis ${ }^{\mathrm{x}}$ antigens or pseudo-Lewis' in the cercaria of the parasite 'target' DC-SIGN to 'instruct' DCs to stimulate the production of $\mathrm{T}_{\mathrm{H}} 2$ responses ${ }^{33}$. In contrast, viral capture through high-mannose HIV-1 gp120 by langerin on Langerhans cells leads to viral elimination, whereas DC-SIGN on dermal dendritic cells enhances HIV-1 infectivity ${ }^{34-37}$. The C-type lectin MGL, with specificity for terminal $\alpha$ - or $\beta$-GalNAc ${ }^{16}$, can recognize pathogens such as filovirus ${ }^{38}$ and helminth parasites such as $S$. mansoni that express terminal GalNAc moieties ${ }^{16}$.

Over 20 pathogenic microorganisms are known to have evolved the capacity to extract sialic acids from their hosts and incorporate these moieties into their own glycoconjugates. Sialylation of glycoconjugates in these pathogens seems to be crucial for their survival in the mammalian hosts, possibly serving as molecular mimics of the host cell 
surfaces to avoid immune attack-by silencing the alternative complement pathway activation, by resisting phagocytosis or by engaging inhibitory siglecs. Siglec-1, Siglec-5 and Siglec-7 have been shown to be involved in host-pathogen interactions of pathogens such as porcine reproductive and respiratory syndrome virus ${ }^{4}$, Neisseria meningitidis, Campylobacter jejuni, Trypanosoma cruzi and Streptococcus group B ${ }^{4,39}$; and pathogen binding to Siglec-1 leads to enhanced pathogen endocytosis and uptake by macrophages (Fig. 2). In addition, Siglec-H, expressed on mouse plasmacytoid DCs, has a role in viral capture and antiviral immunity, as it has been shown to function as an endocytic receptor that internalizes antigen for presentation to $\mathrm{T} \mathrm{cells}^{40}$. Furthermore, Siglec-F and Siglec-G show inhibitory effects on the activation of T and B cells, respectively ${ }^{41}$, suggesting their contribution to negative regulation of adaptive immune responses.

Galectins may also influence immunity by interacting with $\beta$-galactoside-enriched glycoconjugates present in several pathogens ${ }^{8}$. Although the nature of these interactions is still not well characterized, galectin oligomerization and/or lattice formation are likely to play a role ${ }^{42,43}$. For example, galectin-3 binds the lipophosphoglycan of Leishmania major, and this binding leads to the proteolytic removal of the galectin-3 Nterminal domain, preventing oligomerization and lattice formation; galectin- 9 also recognizes L. major by binding to lipophosphoglycan, and this binding promotes glycan-dependent $L$. major-macrophage interactions ${ }^{42}$. Furthermore, galectin-3 acts as a pathogen recognition receptor for LacdiNAc-containing glycans present in helminth parasites ${ }^{43}$. In fact, galectins have been proposed to act as 'alarmins' by sensing danger signals and converting them into activation signals for innate immune cells ${ }^{44}$. Galectin-3-mediated ligand clustering triggers neutrophils to phagocytose, produce reactive oxygen species, release proteases and secrete the chemokine IL-8 (ref. 44). In addition, galectin-3 expression can also alter histamine release and IL-4 synthesis by mast cells ${ }^{45}$. In contrast, galectin-1 has been shown to contribute to resolution of the acute inflammatory response, as it inhibits mast cell degranulation, blocks neutrophil recruitment to sites of inflammation and promotes phosphatidylserine exposure, thus preparing neutrophils for rapid apoptosis ${ }^{46-48}$. Moreover, at the interface of innate and adaptive immunity, galectin-9 binding to specific glycoproteins 'favors' the maturation of human monocyte-derived DCs ${ }^{49}$, and expression of galectin-1 upregulates a substantial subset of genes related to DC migration $^{50}$. In addition, expression of galectin- 1 and/or galectin- 3 can mediate alternative activation of macrophages, which is clearly illustrated by the inability of IL-4 to induce alternative macrophage activation in L gals $^{-1-}$ (galectin-3-deficient) mice ${ }^{51}$ and the ability of galectin-1 signaling to 'skew' L-arginine metabolism toward increased arginase activity in peritoneal macrophages ${ }^{52}$. Partridge and colleagues proposed a glycosylation-dependent mechanism by which galectin-3 cross-linking to complex $\mathrm{N}$-glycans on cytokine receptors may delay their cell surface removal by endocytosis and promote sustained cytokine signaling on leukocytes ${ }^{53}$.

Thus, galectins work as versatile molecules capable of modulating the physiology of different innate immune cells by acting either as cytokines, adhesion molecules or modulators of the immunological synapse. In contrast, most C-type lectins and Siglec-1 are antigen-uptake receptors that facilitate MHC-restricted antigen presentation to T cells. All of these lectins are considered powerful signaling molecules that positively or negatively instruct DC differentiation and subsequent T cell responses.

\section{Homeostasis of adaptive immune responses}

Identification of endogenous factors that control immune tolerance and homeostasis is a key challenge in the field of immunology. Most

\section{Lymphocyte homeostasis}

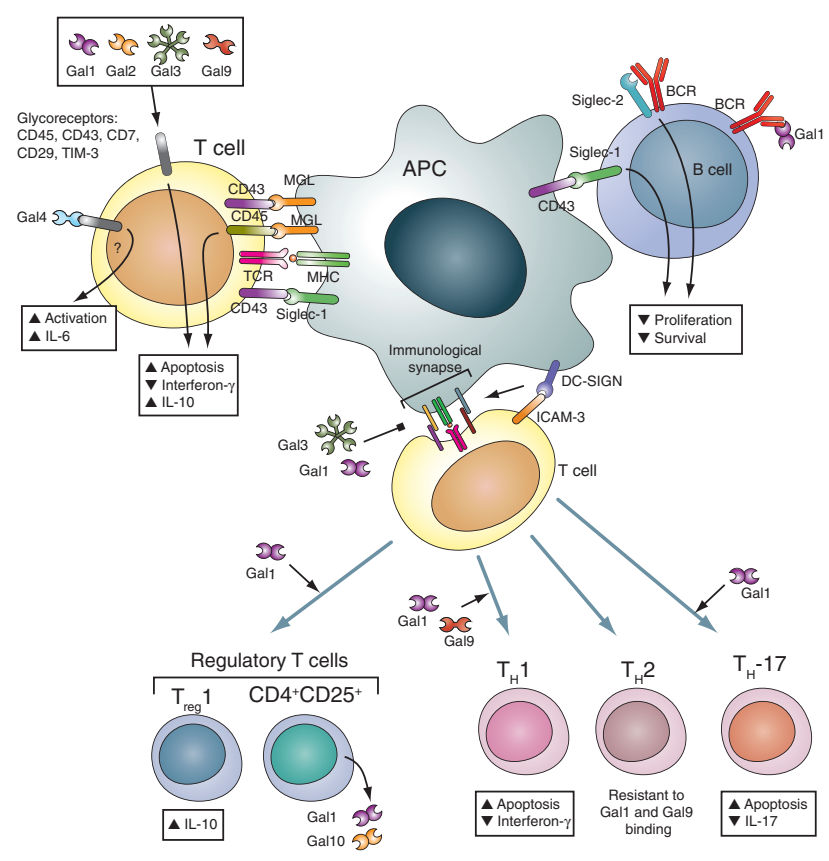

Figure 3 Lectin-glycan interactions in the control of immune cell homeostasis. Lectins are involved in the modification of various cellular interactions owing to the differential glycosylated state of particular counter-receptors. Many studies have shown inhibitory control mechanisms on effector T cells, such as induction of apoptosis by galectin binding to glycosylated CD45, CD43 or TIM-3 or action through C-type lectin-glycan interactions including MGL-CD45 interactions that take place between tolerogenic APCs and effector T cells. Certain glycoreceptors (CD43, CD45, MUC-1) can be recognized by C-type lectins, galectins and siglecs, depending on their glycosylation status. Synapse formation between APCs and T cells can be regulated by galectin- 3 and by DC-SIGN and ICAM-3. APC and B cell function can be altered by Siglec-1, Siglec-2 and galectin1 , thus affecting BCR signaling. In particular, galectin-1 can induce IL-10 production, promote the expansion of regulatory $T$ cells and induce apoptosis of $T_{H} 1$ and $T_{H^{-}} 17$ effectors through specific glycan recognition, thus favoring the development of $\mathrm{T}_{\mathrm{H}} 2$ responses. Gal, galectin.

immune cells are heavily sialylated, which often shields the glycans from recognition by CLRs. However, when cells modify their sialylation pattern, glycans are exposed to allow new CLR-ligand interactions. In this regard, DC-T cell synapse formation can be regulated by DC-SIGN and the adhesion molecule ICAM-3, which is present on a minor population of naive $\mathrm{T}$ cells ${ }^{35}$. In addition, DC-SIGN can interact with endothelial Lewis ${ }^{\mathrm{y}}$-expressing ICAM-2, which is controlled by the fucosyltranferase FUT1 (Fig. 3 and Supplementary Fig. 1 online). Small interfering RNA 'silencing' of FUT1 results in reduced migration and reduced rolling of immature DC on endothelial cells ${ }^{54}$. Neutrophils that express high levels of Lewis ${ }^{\mathrm{x}}$ (CD15) glycans show enhanced binding to immature DC through DC-SIGN ${ }^{55}$.

Cellular interactions between neutrophils and immature DCs have an important function in the transition from innate to adaptive immune responses, whereby activated neutrophils 'instruct' DCs to mature and then to 'trigger' $\mathrm{T}$ cell signaling. In particular, Lewis ${ }^{\mathrm{x}}$ on both MAC-1 $\left(\alpha_{\mathrm{M}} \beta_{2}\right.$ integrin) and CEACAM-1 (carcinoembryonic antigen-related cell adhesion molecule-1) on neutrophils functions as a 'ligand' for DC-SIGN ${ }^{55}$. This highlights the fact that the cell-specific glycosylation machinery can differentially affect recognition by carbohydrate-binding 
proteins (Fig. 3 and Supplementary Fig. 1). In fact, neutrophils contain high expression of FUT-9, which is involved in the biosynthesis of specific Lewis ${ }^{\mathrm{x}}$ glycans. Another cell-specific ligand for DC-SIGN recently identified is the B7 costimulatory molecule-related BTN2A1 (Supplementary Table 1). Also, the C-type lectin MGL, primarily expressed on tolerogenic DCs, has been shown to interact with a subset of $\mathrm{CD}^{+}$and/or CD8 ${ }^{+}$effector T cells ${ }^{16,56}$. The MGL ligand on these $\mathrm{T}$ cells was identified as CD45, which exposes terminal GalNAc (Tn) structures for recognition by MGL. More importantly, binding of MGL to CD45 triggers the phosphatase activity of CD45 that results in enhanced apoptosis of $\mathrm{T}$ cells and reduced secretion of proinflammatory cytokines ${ }^{56}$.

As soluble lectins, galectins can also modulate immune effector functions by controlling cell activation, cytokine synthesis and viability through cross-linkage of glycoreceptors on the surfaces of $\mathrm{T}$ and $\mathrm{B}$ cells $^{8}$. Galectins 1,2,3 and 9 can bind distinct cell-surface glycoprotein receptors and signal different intracellular pathways to modulate $\mathrm{T}$ cell survival and homeostasis ${ }^{57-60}$. Although many glycoproteins contain substantial amounts of lactosamine-containing glycoconjugates, different members of the galectin family bind to a restricted set of $\mathrm{T}$ cell surface glycoproteins (that is, CD43, CD45, CD7, CD29, CD71, TIM$3)^{57-60}$. However, what ultimately controls galectin binding to the surface of T cells is the activity of different glycosyltransferases creating or masking specific ligands for these endogenous lectins ${ }^{61,62}$. Likewise, formation of immune synapses between pre-B and stromal cells, which triggers signal transduction from the pre-B cell receptor (pre-BCR), is galectin-1-dependent ${ }^{63}$.

Sialic acid-dependent siglec-1 interactions can regulate binding to mucin-like molecules that are covered with high densities of sialylated O-linked glycans. As such, mucin (MUC)-1 and CD43 have been identified as specific ligands for Siglec-1; these receptors can mediate cellular contact between T cells and Siglec-1-expressing macrophages ${ }^{4}$. CD22 (Siglec-2) is also a well-documented regulator of B cell signaling, homeostasis and survival ${ }^{64-66}$, and helps set the threshold for antigen-induced B cell activation ${ }^{4}$. B cell receptor ligation leads to increased phosphorylation of the cytoplasmic tail of CD22, which recruits phosphatases (for example, SHP1) to downmodulate BCR signaling. Consistent with these findings, CD22-deficient mice show a hyperimmune response (ref. 67). Cellular activation may reduce the masking and cis-ligand occupation by desialylation to expose siglecs for trans interactions, allowing colocalization of BCR-CD22 complexes in rafts ${ }^{64,68,69}$ (Fig. 3). In addition, many members that belong to the CD33-related siglecs (Siglecs 3, 5, 6, 7, $8,9,10,11$ and 14) are expressed by mature cells of the innate immune system, including neutrophils, eosinophils, macrophages, DCs and NK cells ${ }^{4}$. They have been described to inhibit cellular proliferation, induce apoptosis and block cellular activation; these effects are probably attributable to ITIM motifs present in the siglec cytoplasmic tails ${ }^{4}$.

\section{Protein-glycan interactions in inflammation and autoimmunity}

In several inflammatory autoimmune diseases, such as systemic lupus erythematosus, effector $\mathrm{T}$ cell populations with altered $\mathrm{T}$ cell glycosylation, especially those exposing terminal GalNAc and Gal- $\beta(1-4)-$ GlcNAc structures, are highly upregulated ${ }^{70}$. Both MGL and galectins interact with these structures and have been proven to downmodulate T cell antigen receptor (TCR)-mediated signaling and affect the phosphatase function of CD45 (ref. 56). Indeed, several indications hint at an involvement of galectins in suppression of chronic inflammation. Treatment with galectin-1 suppresses chronic inflammation in several models of autoimmunity by skewing the balance of the immune response toward a $\mathrm{T}_{\mathrm{H}} 2$ cytokine profile $e^{8,71-73}$. In particular, $\mathrm{T}_{\mathrm{H}} 1$ and $\mathrm{T}_{\mathrm{H}^{-17}}$ effector cells express the repertoire of cell surface glycans that are essential for galectin-1 binding and subsequent cell death. By contrast, $\mathrm{T}_{\mathrm{H}} 2$ cells are resistant to galectin- 1 binding through differential $\alpha(2-6)$ sialylation of cell surface glycoproteins ${ }^{73}$. Accordingly, galectin-1-deficient $\left(\right.$ Lgals $\left.^{-/-}\right)$mice develop greater antigen-specific $\mathrm{T}_{\mathrm{H}} 1$ and $\mathrm{T}_{\mathrm{H}^{-17}}$ responses than do wild-type mice ${ }^{73}$. Similarly to galectin-1, galectin-2 induces apoptosis of activated $\mathrm{T}$ cells and ameliorates inflammatory bowel disease ${ }^{58}$; and, in addition, galectin- 9 acts as a specific binding partner of TIM-3, a $\mathrm{T}_{\mathrm{H}} 1$-specific receptor and selectively dampens $\mathrm{T}_{\mathrm{H}} 1$ responses in vivo in a model of autoimmune neuro-inflammation ${ }^{60}$. However, recent evidence indicates that engagement of TIM-3 by galectin-9 can also favor tissue inflammation by synergizing with TLR on $\mathrm{DCs}^{74}$. In this regard, galectin- 4 , another tandem-repeat galectin, contributes to exacerbated inflammation by perpetuating $\mathrm{CD} 4^{+} \mathrm{T}$ cell activation and increasing IL- 6 secretion $^{75}$. Galectin- 1 also contributes to immune tolerance by enhancing IL-10 production $^{76}$ and fostering the expansion of IL-10-producing regulatory T cells in mouse models of autoimmunity, fetal loss and cancer ${ }^{71,77,78}$. Thus, galectin-1 halts inflammatory and autoimmune responses by modulating the $\mathrm{T}_{\mathrm{H}} 1-\mathrm{T}_{\mathrm{H}} 2$ cytokine balance, by inducing $\mathrm{T}$ cell apoptosis and by facilitating the expansion of IL-10-secreting regulatory T cells. Notably, galectin-1 and galectin-10 are overexpressed in $\mathrm{CD} 4{ }^{+} \mathrm{CD} 25^{+}$regulatory $\mathrm{T}$ cells and have been reported to be essential for the immunosuppressive activity of these cells ${ }^{79,80}$.

Notably, multivalent galectin-3-N-glycan lattices can limit TCR clustering at sites of immunological synapse and increase agonist threshold for TCR signaling, and thus they may contribute to T cell homeostasis ${ }^{81}$. Galectin-1 can also interfere with TCR signaling by inducing partial TCR- $\zeta$ chain phosphorylation, thus antagonizing $\mathrm{T}$ cell functions such as $\mathrm{T}$ cell proliferation and IL-2 secretion that require sustained signal transduction $^{82}$. Galectin-carbohydrate lattices can therefore control T cell-mediated inflammation through multiple mechanisms, affecting the survival, signaling and functionality of effector and regulatory cells.

C-type lectins also play a role in the modulation of inflammatory and autoimmune diseases. In vivo, targeting a central nervous system peptide to the CLR DEC205 on immature mouse DCs induces tolerance in experimental autoimmune encephalomyelitis (EAE) ${ }^{25}$. Similarly, mannosylated myelin proteolipid protein peptides can inhibit the onset of EAE in mice, probably by targeting the mannose receptor on immature DCs, whereas unglycosylated peptides induce severe EAE ${ }^{83,84}$. These findings indicate that the natural function of CLRs as antigen uptake receptors is associated with the maintenance of tolerance for homeostatic control ${ }^{26}$. In contrast, certain siglecs, such as Siglec-1 (sialoadhe$\sin$ ), are upregulated by inflammatory macrophages; for example, in proliferative glomerulonephritis, the expression of Siglec-1 correlates with proteinuria ${ }^{85}$. At the same time, Siglec- 1 may modulate T cell function and activation during immune responses, as sialoadhesin-deficient mice show reduced inflammation owing to reduced influx of macrophages and $\mathrm{T}$ cells to inflammatory sites ${ }^{4}$.

\section{Protein-glycan interactions in tumor immunity}

In recent years, many laboratories have made considerable efforts to identify a 'poor prognosis signature' of tumor- and metastasis-associated genes and to predict the clinical outcome of neoplastic disease. Changes in glycosylation have been shown to strongly associate with the development of cancer and metastasis ${ }^{86}$. This differential glycosylation of cancerous and healthy tissues is often restricted to altered glycan expression of tumor cells or their secreted glycoproteins. In many cases, the structural changes have been further correlated with changes in the activity of one or more glycosyltransferases during the process of transformation from normal to tumor cells ${ }^{86}$ (Supplementary Fig. 1). Changes in glycosylation could result in loss of cell adhesion, an event associated with 


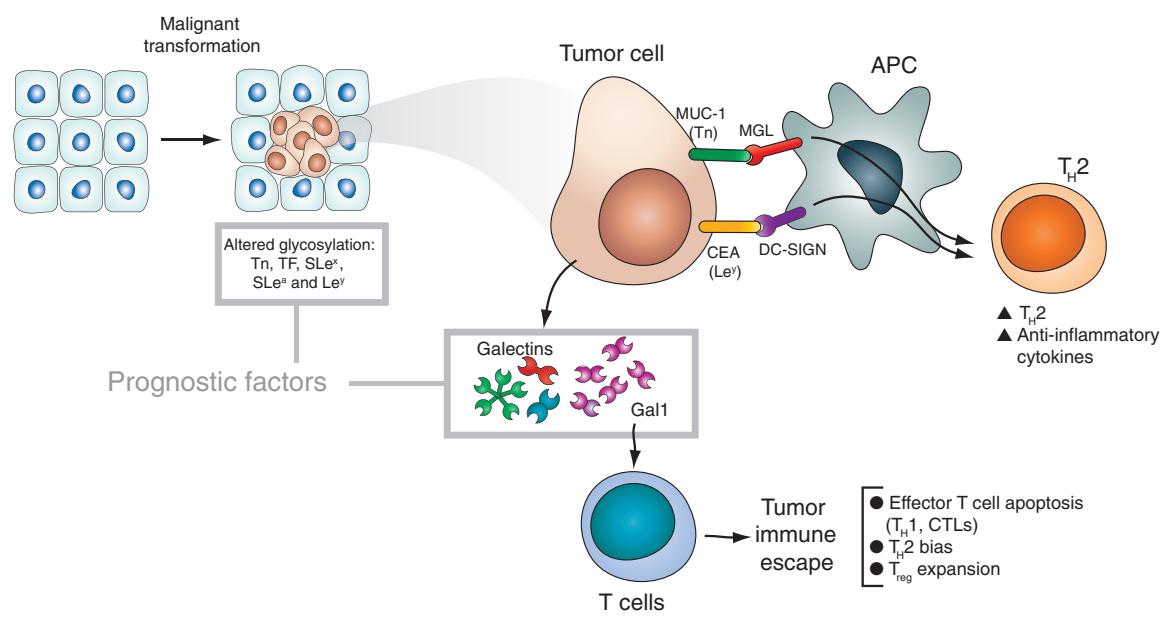

Figure 4 Glycan modifications during tumor development results in selective recognition by lectins and modulation of immune responses. Malignant transformation often correlates with altered glycosylation of glycoproteins on the surface of tumor cells. These glycosylation changes, which have been widely used as prognostic markers of disease progression, can be detected by several galectins and C-type lectins (MGL and DC-SIGN). The binding of these lectins to altered glycoproteins may affect APC function, leading to anti-inflammatory responses. Alternatively, galectins synthesized by the tumor may target the survival and proliferation of effector $\mathrm{T}$ cells, skew the balance toward $\mathrm{a} \mathrm{T}_{\mathrm{H}} 2$ cytokine profile, and/or induce the expansion of regulatory $T$ cells to favor immune escape by the tumor. CEA, carcinoembryonic antigen; Le, Lewis; SLe, sialylated Lewis; TF, Thomsen-Friedenreich disaccharide.

increased cell invasiveness of primary tumors to distant sites. Notably, the metastatic potential of some tumor cells has been extensively correlated with increased sialylation of cell surface glycoproteins, which is consistent with the known ability of sialic acid-binding lectins such as selectins to mediate cell adhesion and extravasation during the metastatic process (Fig. 4 and Supplementary Fig. 1) ${ }^{87-89}$. Also, loss of disialyl Lewis ${ }^{a}$ structures (ligands for Siglec-7), an occurrence associated with increased sialyl Lewis ${ }^{\mathrm{a}}$ (ligands for E-selectin) in human colon cancers, induces loss or gain of function of lectin receptors ${ }^{90,91}$. In addition, several carbohydrate structures, such as Tn (GalNAc- $\alpha$-Ser/Thr, CD175), sialyl Tn ( $N$-acetylneuraminic acid- $\alpha 6-G a l N A c-\alpha-S e r / T h r, C D 175 s)$, Thomsen-Friedenreich disaccharide (Gal- $\beta(1-3)$-GalNAc, CD176) and (sialylated) Lewis antigens (CD15s) are highly upregulated in malignant cells and have been widely used as diagnostic and prognostic markers ${ }^{87}$. Monoclonal antibodies to these glycan epitopes have been used for the detection of tumor growth and metastasis, to monitor disease status and the effectiveness of different therapies.

The recent finding that several of these tumor-related glycoforms of carcinoembryonic antigen ${ }^{88}$ and MUC-1 (ref. 89) are specific ligands for CLRs expressed on DCs, such as DC-SIGN and MGL, opens up a new area of exploring whether these specific glycoforms affect CLR signaling and DC differentiation, thereby modulating innate and adaptive antitumor responses. Expression of Tn epitopes on MUC-1 is associated with poor prognosis in several types of cancer ${ }^{86}$; of note, Tn glycans on MUC-1 bind MGL and instruct DC to drive $\mathrm{T}_{\mathrm{H}} 2$-mediated responses, which, unlike those of $\mathrm{T}_{\mathrm{H}} 1$ effector cells, do not contribute to tumor eradication. These findings illustrate the relevance of altered glycosylation in the tumor microenvironment in the impaired function of DCs. Studies in vitro and in vivo in both mice and human have demonstrated that high-affinity binding structures, such as antibodies and glycans, may be used to 'direct' vaccine vehicles to CLRs on DC and thus stimulate antigen-specific $\mathrm{T}$ cell responses. For example, the $\mathrm{C}$-type lectins langerin and DC-SIGN are expressed on LC and DC and have been successfully targeted for the subsequent generation of tumor-specific immunity 92,93 . Targeting CLRs thus may not only provide the DC-specific target but also may simultaneously facilitate antigen internalization for MHC- or CD1-mediated antigen presentation.

In addition to altered glycosylation, gene and protein expression profiles of tumors have repeatedly led to the identification of galectins as proteins that are expressed in a plethora of tumors and metastatic lesions ${ }^{9}$. Galectins can certainly influence tumor progression through many mechanisms, including modulation of neoplastic transformation, tumor cell survival, angiogenesis and tumor metastasis 9 . Secretion of galectin-1 has been shown to contribute to the immunosuppressive activity of melanoma, Hodgkin's lymphoma and prostate cancer cells, suggesting the potential involvement of this protein in immune escape by tumors ${ }^{78,94,95}$. Hence, it is assumed that the galectin repertoire in the tumor microenvironment and the glycosylation pattern of inflammatory cells might be important in cancer progression and immuno-editing.

\section{Differential glycosylation control innate and adaptive responses}

As highlighted above, glycans have enormous structural and functional diversity and are critical in a variety of biological processes, including those that are fundamental for the development and homeostasis of the immune response ${ }^{1,96}$. Most immune processes, including activation, differentiation and homing, are accompanied by a programmed remodeling of cell surface glycans, which themselves are the products of a repertoire of glycosyltransferases and glycosidases, acting sequentially and dictating the glycosylation 'signature' of each effector cell type ${ }^{96-99}$. The function of decoding the biological information encrypted by this glycosylation 'signature' is assigned in part to endogenous glycan-binding proteins or lectins (that is, C-type lectins, galectins and siglecs), whose expression and function are regulated at sites of inflammation ${ }^{96}$. A recent approach to decipher the 'glycomics' of the immune system revealed extensive changes, which critically affect the binding and signaling of glycan-binding proteins, in the expression of $\mathrm{N}$ - and $\mathrm{O}$-glycans during T-cell activation ${ }^{97,98}$, T helper differentiation ${ }^{73,98}$ and DC maturation ${ }^{99}$. In addition, changes in glycosylation are often hallmark of the transition from normal to inflamed or neoplastic tissue, providing new types of opportunities for therapeutic interventions ${ }^{87}$.

In recent years, many gene knockout experiments have facilitated our understanding of the impact of O- and N-glycosylation pathways in immune cell function. Demetriou and colleagues found that targeted deletion of the locus encoding $N$-acetylglucosaminyltransferase-5, resulting in lack of $\beta(1-6)$ branching of tri- and tetra-antennary complex Nglycans, ligands for galectin-3, enhanced T cell receptor (TCR)-mediated signaling and induced 'hyper- $\mathrm{T}_{\mathrm{H}} 1$ ' responses and greater susceptibility to autoimmune disease $\mathrm{e}^{81,98}$, which was the result of a restricted TCR aggregation by binding to galectins in the immunological synapse. The authors recently extended these findings showing that metabolite supply of the hexosamine pathway restores the high-affinity ligands for galectins, which suppresses TCR signaling and proliferation, inhibits $\mathrm{T}_{\mathrm{H}} 1$ skewing and prevents the development of autoimmune inflammation ${ }^{100,101}$. Furthermore, it has been reported that the lack of polylactosamine 
renders T cells, B cells and macrophages more sensitive to agonist-induced activation $^{102}$. Thus, lattice formation after the binding of complex N-glycan to galectins effectively traps glycoprotein receptors at the cell surface and restricts cells' responsiveness to receptor agonists.

In addition to their contribution to $\mathrm{T}$ cell homeostasis and receptor turnover, a recent study highlighted a new, unpredicted role of $\mathrm{N}$-glycans at the interface of innate immunity and autoimmune inflammation: this study described the development of a systemic lupus erythematosus-like syndrome in mice deficient in the enzyme $\alpha$-mannosidase II $(\alpha M-I I)$ that seems to be driven exclusively through a mechanism involving innate immunity ${ }^{103}$. The authors provided evidence that aberrantly expressed mannose-containing glycans in $\alpha \mathrm{M}$-II null mice can elicit an innate immune response mediated by the C-type lectin MBL or mannose receptor ${ }^{103}$. These findings underscore a critical role for the mannose recognition system, classically exploited by pathogens to trigger innate immunity, in the initiation of organ-specific autoimmunity.

As with the $\mathrm{N}$-glycan studies, recent efforts involving genetic manipulation of the O-glycosylation pathway have revealed essential roles of O-glycans in immune system homeostasis ${ }^{96} . \alpha(2-3)$-Sialyltransferase I catalyzes sialic acid incorporation into core $1 \mathrm{O}$-glycans during protein O-glycosylation ${ }^{104}$. Deletion of the St3gal1 locus, encoding this enzyme, yields constitutive exposure of unsialylated core $1 \mathrm{O}$-glycans, which were found to be associated with increased $\mathrm{CD}^{+} \mathrm{T}$ cell apoptosis and contraction of the $\mathrm{CD} 8^{+} \mathrm{T}$ cell compartment upon microbial challenge ${ }^{104}$. Hence, differential sialylation of cell surface glycoproteins may serve as an 'on-off' switch that controls the decisions between immune cell responsiveness or tolerance ${ }^{96,105}$. Notably, it has been reported that the differential $\alpha(2-6)$ sialylation of the Fc region can control the proor anti-inflammatory effects of immunoglobulin $\mathrm{G}$ by modulating its binding to $\mathrm{Fc} \gamma$ receptors ${ }^{105}$. In addition, aberrant glycosylation of immunoglobulins has been linked to the pathogenesis of diseases such as rheumatoid arthritis and immunoglobulin A nephropathy ${ }^{106,107}$. In particular, levels of agalactosyl and asialo glycoforms of immunoglobulin G have been shown to correlate with disease activity in rheumatoid arthritis in humans and in experimental autoimmune models ${ }^{106}$. Thus, differential sialylation can have a decisive role in the regulation of diverse immunological processes associated with immune cell tolerance and inflammation.

O-glycans are also the chief components of the intestinal mucus layer that covers the gastrointestinal epithelium and protects mucosal tissue from potentially harmful pathogens. It has been recently found that deficiency of the core 3 transferase $\mathrm{C} 3 \mathrm{GnT}$ results in elimination of core 3-derived O-glycans and presentation of naked Tn antigen in mouse colonic epithelium ${ }^{108}$. C3GnT-deficient mice have an increased susceptibility to inflammatory bowel disease and colorectal adenocarcinoma ${ }^{108}$, suggesting that core 3-derived O-glycans are key components of intestinal mucin and that disruption of these structures is associated with the initiation and perpetuation of intestinal inflammation. Notably, altered O-glycans are seen in more than $90 \%$ of colorectal cancers, which are strongly associated with ulcerative colitis. Thus, altered expression of glycosyltransferases would seem to modulate the glycosylation pattern of epithelial or immune cells and contribute to human pathology.

In this regard, a somatic mutation in $\operatorname{Cos} m c$, a gene that encodes a molecular 'chaperone' that is required for proper folding and activity of the T-synthase (core $1 \beta(1-3)$-galactosyltransferase), was recently reported ${ }^{107}$. This mutation creates a truncated $\mathrm{O}$-glycan composed of only $\mathrm{N}$-acetylgalactosamine (Tn antigen); this seems to be the cause of a rare autoimmune disease called 'Tn syndrome', in which subpopulations of blood cells in all lineages carry an incompletely glycosylated membrane glycoprotein, as well as of other Tn-related syndromes such as immunoglobulin A nephropathy ${ }^{107}$. Thus, modifications on terminal
$\mathrm{N}$ - and $\mathrm{O}$-glycans provide distinct and essential contributions to innate and adaptive immune responses, with profound implications for the initiation and perpetuation of immune-mediated disorders.

\section{Conclusions and future directions}

Advances in deciphering the information encoded by the 'glycome'-the entire repertoire of complex sugar structures expressed in cells and tissues-has created much interest in its potential biotechnological and pharmaceutical applications, but progress has been hampered by the inherent difficulties in studying the structure-function relationship of these complex molecules. However, innovative developments, including glycoarray technology and frontal affinity chromatography $7,14,109$, are rapidly changing the scene and creating opportunities for real progress over the next few years.

Recent efforts toward decoding the information contained in protein-saccharide interactions (Supplementary Table 1) have illuminated essential roles of glycan-binding proteins or lectins (that is, galectins, C-type lectins and siglecs) in host-pathogen interactions, initiation of innate immunity and modulation of immune tolerance and homeostasis. However, we are just beginning to understand the powerful contribution of protein-glycan interactions to the control of immune tolerance versus immunity, thus predicting a role for endogenous lectins and their specific saccharide ligands as potential targets for effective treatment of chronic inflammation, autoimmunity and cancer. Before these therapeutic strategies can be fully realized, however, there is still much to be learned concerning the contribution of glycan specificity and the nature of protein-glycan lattices in the amplification, silencing or tuning of innate and adaptive immune processes.

Note: Supplementary information is available on the Nature Immunology website.

\section{ACKNOWLEDGMENTS}

We apologize to the many authors whose papers could not be cited owing to space limitations. We thank all members of the Y.v.K. and G.A.R. laboratories for help and discussion, especially J.J. Garcia-Vallejo, S. van Vliet, M.A. Toscano, J.M. Ilarregui and G. Bianco; and H.F. Rosenberg, I. van Die and T.B.H. Geijtenbeek for critical reading of the manuscript. Supported by grants from Dutch Scientific Organization (NWO/ZONMW), Multiple Sclerosis Research Foundation the Netherlands, Association of International Cancer Research, VU Medical Centre Institute of Cancer and Immunology (VICI) and Bridging Grant Consortium for Functional Glycomics (Y.v.K.) and by The Cancer Research Institute "Elaine R. Shepard" Award, Fundación Sales, Agencia Nacional de Promoción Científica y Tecnológica, University of Buenos Aires and Consejo Nacional de Investigaciones Científicas y Tecnológicas (G.A.R.).

Published online at http://www. nature.com/natureimmunology Reprints and permissions information is available online at http://npg.nature.com/ reprintsandpermissions/

1. Ohtsubo, K. \& Marth, J.D. Glycosylation in cellular mechanisms of health and disease. Cell 126, 855-867 (2006).

2. van Die, I. \& Cummings, R.D. Glycans modulate immune responses in helminth infections and allergy. Chem. Immunol. Allergy 90, 91-112 (2006).

3. Leffler, H., Carlsson, S., Hedlund, M., Qian, Y. \& Poirier, F. Introduction to galectins. Glycoconj. J. 19, 433-440 (2004).

4. Crocker, P.R., Paulson, J.C. \& Varki, A. Siglecs and their roles in the immune system. Nat. Rev. Immunol. 7, 255-266 (2007).

5. Robinson, M.J., Sancho, D., Slack, E.C., LeibundGut-Landmann, S. \& Reis e Sousa, C. Myeloid C-type lectins in innate immunity. Nat. Immunol. 7, 1258-1265 (2006).

6. Weis, W.I., Taylor, M.E. \& Drickamer, K. The C-type lectin superfamily in the immune system. Immunol. Rev. 163, 19-34 (1998)

7. Hirabayashi, J. et al. Oligosaccharide specificity of galectins: a search by frontal affinity chromatography. Biochim. Biophys. Acta 1572, 232-254 (2002).

8. Rabinovich, G.A., Toscano, M.A., Jackson, S.S. \& Vasta, G.R. Functions of cell sur face galectin-glycoprotein lattices. Curr. Opin. Struct. Biol. 17, 513-520 (2007).

9. Liu, F.T. \& Rabinovich, G.A. Galectins as modulators of tumour progression. Nat Rev. Cancer 5, 29-41 (2005).

10. Nieminen, J., Kuno, A., Hirabayashi, J. \& Sato, S. Visualization of Galectin-3 oligomerization on the surface of neutrophils and endothelial cells using fluorescence resonance energy transfer. J. Biol. Chem. 282, 1374-1383 (2007). 
11. Zelensky, A.N. \& Gready, J.E. The C-type lectin-like domain superfamily. FEBS J. 272, 6179-6217 (2005).

12. Figdor, C.G., van Kooyk, Y. \& Adema, G.J. C-type lectin receptors on dendritic cells and Langerhans cells. Nat. Rev. Immunol. 2, 77-84 (2002).

13. Appelmelk, B.J. et al. Cutting edge: carbohydrate profiling identifies new pathogens that interact with dendritic cell-specific ICAM-3-grabbing nonintegrin on dendritic cells. J. Immunol. 170, 1635-1639 (2003).

14. Blixt, 0 . et al. Printed covalent glycan array for ligand profiling of diverse glycan binding proteins. Proc. Natl. Acad. Sci. USA 101, 17033-17038 (2004).

15. Feinberg, H., Castelli, R., Drickamer, K., Seeberger, P.H. \& Weis, W.I. Multiple modes of binding enhance the affinity of DC-SIGN for high mannose N-linked glycans found on viral glycoproteins. J. Biol. Chem. 282, 4202-4209 (2007).

16. van Vliet, S.J., Saeland, E. \& van Kooyk, Y. Sweet preferences of MGL: carbohydrate specificity and function. Trends Immunol. 29, 83-90 (2008).

17. Brown, G.D. Dectin-1: a signalling non-TLR pattern-recognition receptor. Nat. Rev. Immunol. 6, 33-43 (2006).

18. Rogers, N.C. et al. Syk-dependent cytokine induction by Dectin-1 reveals a novel pattern recognition pathway for C type lectins. Immunity 22, 507-517 (2005).

19. Taylor, M.E. \& Drickamer, K. Paradigms for glycan-binding receptors in cell adhesion. Curr. Opin. Cell Biol. 19, 572-577 (2007).

20. van Kooyk, Y. \& Geijtenbeek, T.B. DC-SIGN: escape mechanism for pathogens. Nat. Rev. Immunol. 3, 697-709 (2003).

21. Blixt, O. \& Collins, B.E., van den Nieuwenhof, I.M., Crocker, P.R. \& Paulson, J.C. Sialoside specificity of the Siglec family assessed using novel multivalent probes: identification of potent inhibitors of myelin-associated glycoprotein. J. Biol. Chem. 278, 31007-31019 (2003)

22. Powell, L.D., Sgroi, D., Sjoberg, E.R., Stamenkovic, I. \& Varki, A. Natural ligands of the $\mathrm{B}$ cell adhesion molecule CD22 beta carry $\mathrm{N}$-linked oligosaccharides with alpha-2,6-linked sialic acids that are required for recognition. J. Biol. Chem. 268, 7019-7027 (1993)

23. Collins, B.E. et al. Masking of CD22 by cis ligands does not prevent redistribution of CD22 to sites of cell contact. Proc. Natl. Acad. Sci. USA 101, 6104-6109 (2004).

24. Barton, G.M. \& Medzhitov, R. Toll-like receptor signaling pathways. Science $\mathbf{3 0 0}$, 1524-1525 (2003)

25. Bonifaz, L.C. et al. In vivo targeting of antigens to maturing dendritic cells via the DEC-205 receptor improves T cell vaccination. J. Exp. Med. 199, 815-824 (2004).

26. Geijtenbeek, T.B., van Vliet, S.J., Engering, A., 't Hart, B.A. \& van Kooyk, Y. Self- and nonself-recognition by C-type lectins on dendritic cells. Annu. Rev. Immunol. 22, 33-54 (2004).

27. Meyer, S. et al. DC-SIGN mediates binding of dendritic cells to authentic pseudoLewis $^{Y}$ glycolipids of Schistosoma mansoni cercariae, the first parasite-specific ligand of DC-SIGN. J. Biol. Chem. 280, 37349-37359 (2005).

28. Gringhuis, S.I. et al. C-type lectin DC-SIGN modulates Toll-like receptor signaling via Raf-1 kinase-dependent acetylation of transcription factor NF-кB. Immunity $\mathbf{2 6}$, 605-616 (2007)

29. Hodges, A. et al. Activation of the lectin DC-SIGN induces an immature dendritic cell phenotype triggering Rho-GTPase activity required for HIV-1 replication. Nat. Immunol. 8, 569-577 (2007)

30. Underhill, D.M., Rossnagle, E., Lowell, C.A. \& Simmons, R.M. Dectin-1 activates Syk tyrosine kinase in a dynamic subset of macrophages for reactive oxygen production. Blood 106, 2543-2550 (2005).

31. LeibundGut-Landmann, S. et al. Syk- and CARD9-dependent coupling of innate immunity to the induction of $\mathrm{T}$ helper cells that produce interleukin 17. Nat. Immunol. 8, 630-638 (2007).

32. Bergman, M., Del Prete, G., van Kooyk, Y. \& Appelmelk, B. Helicobacter pylori phase variation, immune modulation and gastric autoimmunity. Nat. Rev. Microbiol. 4, 151-159 (2006).

33. van Liempt, E. et al. Schistosoma mansoni soluble egg antigens are internalized by human dendritic cells through multiple C-type lectins and suppress TLR-induced dendritic cell activation. Mol. Immunol. 44, 2605-2615 (2007).

34. Stambach, N.S. \& Taylor, M.E. Characterization of carbohydrate recognition by langerin, a C-type lectin of Langerhans cells. Glycobiology 13, 401-410 (2003).

35. Geijtenbeek, T.B. et al. Identification of DC-SIGN, a novel dendritic cell-specific ICAM-3 receptor that supports primary immune responses. Cell 100, 575-585 (2000).

36. Geijtenbeek, T.B. et al. DC-SIGN, a dendritic cell-specific HIV-1-binding protein that enhances trans-infection of T cells. Cell 100, 587-597 (2000).

37. de Witte, L. et al. Langerin is a natural barrier to HIV-1 transmission by Langerhans cells. Nat. Med. 13, 367-371 (2007).

38. Takada, A. et al. Human macrophage C-type lectin specific for galactose and $\mathrm{N}$ acetylgalactosamine promotes filovirus entry. J. Virol. 78, 2943-2947 (2004).

39. Crocker, P.R. Siglecs in innate immunity. Curr. Opin. Pharmacol. 5, 431-437 (2005)

40. Blasius, A.L. \& Colonna, M. Sampling and signaling in plasmacytoid dendritic cells: the potential roles of Siglec-H. Trends Immunol. 27, 255-260 (2006).

41. Tateno, H. et al. Distinct endocytic mechanism of CD22 (Siglec-2) and Siglec-F reflects roles in cell signaling and innate immunity. Mol. Cell. Biol. 27, 5699-5710 (2007).

42. Pelletier, I. et al. Specific recognition of Leishmania major poly- $\beta$-galactosyl epitopes by galectin-9: possible implication of galectin-9 in interaction between $L$. major and host cells. J. Biol. Chem. 278, 22223-22230 (2003).
43. van den Berg, T.K. et al. LacdiNAc-glycans constitute a parasite pattern for galectin3-mediated immune recognition. J. Immunol. 173, 1902-1907 (2004).

44. Nieminen, J., St-Pierre, C. \& Sato, S. Galectin-3 interacts with naive and primed neutrophils, inducing innate immune responses. J. Leukoc. Biol. 78, 1127-1135 (2005).

45. Chen, H.Y. et al. Role of galectin-3 in mast cell functions: galectin-3-deficient mast cells exhibit impaired mediator release and defective JNK expression. J. Immunol. 177, 4991-4997 (2006).

46. Rabinovich, G.A., Sotomayor, C.E., Riera, C.M., Bianco, I. \& Correa, S.G. Evidence of a role for Galectin-1 in acute inflammation. Eur. J. Immunol. 30, 1331-1339 (2000).

47. La, M. et al. A novel biological activity for galectin-1: inhibition of leukocyte-endothelial cell interactions in experimental inflammation. Am. J. Pathol. 163, 1505-1515 (2003).

48. Stowell, S.R. et al. Human galectin-1, -2 , and -4 induce surface exposure of phosphatidylserine in activated human neutrophils but not in activated T cells. Blood 109, 219-227 (2007)

49. Dai, S.Y. et al. Galectin-9 induces maturation of human monocyte-derived dendritic cells. J. Immunol. 175, 2974-2981 (2005).

50. Fulcher, J.A. et al. Galectin-1-matured human monocyte-derived dendritic cells have enhanced migration through extracellular matrix. J. Immunol. 177, 216-226 (2006).

51. MacKinnon, A.C. et al. Regulation of alternative activation by galectin-3. J. Immunol. $180,2650-2658$ (2008)

52. Correa, S.G. et al. Opposite effects of galectin-1 on alternative metabolic pathways of L-arginine in resident, inflammatory, and activated macrophages. Glycobiology 13 119-128 (2003).

53. Partridge, E.A. et al. Regulation of cytokine receptors by Golgi N-glycan processing and endocytosis. Science 306, 120-124 (2004).

54. Garcia-Vallejo, J.J. et al. DC-SIGN mediates adhesion and rolling of dendritic cells on primary human umbilical vein endothelial cells through Lewis( $Y$ ) antigen expressed on ICAM-2. Mol. Immunol. 45, 2359-2369 (2007).

55. van Gisbergen, K.P., Geijtenbeek, T.B. \& van Kooyk, Y. Close encounters of neutrophils and DCs. Trends Immunol. 26, 626-631 (2005).

56. van Vliet, S.J., Gringhuis, S.I., Geijtenbeek, T.B. \& van Kooyk, Y. Regulation of effector $T$ cells by antigen-presenting cells via interaction of the C-type lectin MGL with CD45. Nat. Immunol. 7, 1200-1208 (2006)

57. Stillman, B.N. et al. Galectin-3 and galectin-1 bind distinct cell surface glycoprotein receptors to induce T cell death. J. Immunol. 176, 778-789 (2006).

58. Paclik, D. et al. Galectin-2 induces apoptosis of lamina propria T lymphocytes and ameliorates acute and chronic experimental colitis in mice. J. Mol. Med. published online, doi:10.1007/s00109-007-0290-2 (7 December 2007).

59. Fukumori, T. et al. CD29 and CD7 mediate galectin-3-induced type II T-cell apoptosis. Cancer Res. 63, 8302-8311 (2003).

60. Zhu, C. et al. The Tim-3 ligand galectin-9 negatively regulates $T$ helper type 1 immunity. Nat Immunol 6, 1245-1252 (2005).

61. Amano, M., Galvan, M., He, J. \& Baum, L.G. The ST6Gal I sialyltransferase selectively modifies N-glycans on CD45 to negatively regulate Galectin-1-induced CD45 clustering, phosphatase modulation, and T cell death. J. Biol. Chem. 278, 7469-7475 (2003).

62. Stowell, S.R. et al. Galectins-1, -2 , and -3 exhibit differential recognition of sialylated glycans and blood group antigens. J. Biol. Chem. 283, 10109-10123 (2008).

63. Rossi, B., Espeli, M., Schiff, C. \& Gauthier, L. Clustering of pre-B cell integrins induces galectin-1-dependent pre-B cell receptor relocalization and activation. J. Immunol. 177, 796-803 (2006).

64. Collins, B.E., Smith, B.A., Bengtson, P. \& Paulson, J.C. Ablation of CD22 in liganddeficient mice restores B cell receptor signaling. Nat. Immunol. 7, 199-206 (2006).

65. Han, S., Collins, B.E., Bengtson, P. \& Paulson, J.C. Homomultimeric complexes of CD22 in B cells revealed by protein-glycan cross-linking. Nat. Chem. Biol. 1, 93-97 (2005).

66. Grewal, P.K et al. ST6Gal-I restrains CD22-dependent antigen receptor endocytosis and Shp-1 recruitment in normal and pathogenic immune signaling. Mol. Cell. Biol. 26, 4970-4981 (2006).

67. Doody, G.M. et al. A role in B cell activation for CD22 and the protein tyrosine phosphatase SHP. Science 269, 242-244 (1995).

68. Hoffmann, A. et al. Siglec-G is a B1 cell-inhibitory receptor that controls expansion and calcium signaling of the B1 cell population. Nat. Immunol. 8, 695-704 (2007).

69. Paulson, J.C., Blixt, O. \& Collins, B.E. Sweet spots in functional glycomics. Nat Chem. Biol. 2, 238-248 (2006).

70. Buzás, E.l. et al. Carbohydrate recognition systems in autoimmunity. Autoimmunity 39, 691-704 (2006)

71. Toscano, M.A. et al. Galectin-1 suppresses autoimmune retinal disease by promoting concomitant Th2- and T regulatory-mediated anti-inflammatory responses. J. Immunol. 176, 6323-6332 (2006).

72. Perone, M.J. et al. Dendritic cells expressing transgenic galectin-1 delay onset of autoimmune diabetes in mice. J. Immunol. 177, 5278-5289 (2006).

73. Toscano, M.A. et al. Differential glycosylation of $T_{H} 1, T_{H} 2$ and $T_{H^{-}} 17$ effector cells selectively regulates susceptibility to cell death. Nat. Immunol. 8, 825-834 (2007).

74. Anderson, A.C. et al. Promotion of tissue inflammation by the immune receptor Tim-3 expressed on innate immune cells. Science $\mathbf{3 1 8}, 1141-1143$ (2007). 
75. Hokama, A. et al. Induced reactivity of intestinal CD4 ${ }^{+} \mathrm{T}$ cells with an epithelial cell lectin, galectin-4, contributes to exacerbation of intestinal inflammation. Immunity 20, 681-693 (2004).

76. van der Leij, J. et al. Strongly enhanced IL-10 production using stable galectin-1 homodimers. Mol. Immunol. 44, 506-513 (2007).

77. Blois, S.M. et al. A pivotal role for galectin-1 in fetomaternal tolerance. Nat. Med. 13, 1450-1457 (2007).

78. Juszczynski, P. et al. The AP1-dependent secretion of galectin-1 by Reed Sternberg cells fosters immune privilege in classical Hodgkin lymphoma. Proc. Natl. Acad. Sci. USA 104, 13134-13139 (2007).

79. Garin, M.I. et al. Galectin-1: a key effector of regulation mediated by $\mathrm{CD} 4{ }^{+} \mathrm{CD} 25^{+} \mathrm{T}$ cells. Blood 109, 2058-2065 (2007).

80. Kubach, J. et al. Human $\mathrm{CD} 4{ }^{+} \mathrm{CD} 25^{+}$regulatory $\mathrm{T}$ cells: proteome analysis identifies galectin-10 as a novel marker essential for their anergy and suppressive function. Blood 110, 1550-1558 (2007).

81. Demetriou, M., Granovsky, M., Quaggin, S. \& Dennis, J.W. Negative regulation of T-cell activation and autoimmunity by Mgat5 N-glycosylation. Nature 409, 733-739 (2001)

82. Chung, C.D., Patel, V.P., Moran, M., Lewis, L.A. \& Miceli, M.C. Galectin-1 induces partial TCR $\zeta$-chain phosphorylation and antagonizes processive TCR signal transduction. J. Immunol. 165, 3722-3729 (2000).

83. Kel, J. et al. Soluble mannosylated myelin peptide inhibits the encephalitogenicity of autoreactive T cells during experimental autoimmune encephalomyelitis. Am. J. Pathol. 170, 272-280 (2007).

84. Luca, M.E. et al. Mannosylated PLP(139-151) induces peptide-specific tolerance to experimental autoimmune encephalomyelitis. J. Neuroimmunol. 160, 178-187 (2005)

85. York, M.R. et al. A macrophage marker, Siglec-1, is increased on circulating monocytes in patients with systemic sclerosis and induced by type I interferons and toll-like receptor agonists. Arthritis Rheum. 56, 1010-1020 (2007).

86. Hakomori, S. Glycosylation defining cancer malignancy: new wine in an old bottle. Proc. Natl. Acad. Sci. USA 99, 10231-10233 (2002).

87. Dube, D.H. \& Bertozzi, C.R. Glycans in cancer and inflammation-potential for therapeutics and diagnostics. Nat. Rev. Drug Discov. 4, 477-488 (2005).

88. Aarnoudse, C.A., Garcia-Vallejo, J.J., Saeland, E. \& van Kooyk, Y. Recognition of tumor glycans by antigen presenting cells. Curr. Opin. Immunol. 18, 105-111 (2006).

89. Saeland, E. et al. The C-type lectin MGL expressed by dendritic cells detects glycan changes on MUC1 in colon carcinoma. Cancer Immunol. Immunother. 56, 1225-1236 (2007).

90. Kawamura, Y.I. et al. Introduction of Sda carbohydrate antigen in gastrointestinal cancer cells eliminates selectin ligands and inhibits metastasis. Cancer Res. 65, 6220-6227 (2005).

91. Miyazaki, K. et al. Loss of disialyl Lewis ${ }^{\text {a }}$, the ligand for lymphocyte inhibitory receptor sialic acid-binding immunoglobulin-like lectin-1 (Siglec-7) associated with increased sialyl Lewis ${ }^{a}$ expression on human colon cancers. Cancer Res. 64, 4498-4505
(2004).

92. Tacken, P.J., de Vries, I.J.M., Torensma, R. \& Figdor, C.G. Dendritic-cell immunotherapy: from ex vivo loading to in vivo targeting. Nat. Rev. Immunol. 7, 790-802 (2007).

93. Idoyaga, J. et al. Langerin/CD207 receptor on dendritic cells mediates efficient antigen presentation on MHC I and II products in vivo. J. Immunol. 180, 3647-3650 (2008).

94. Rubinstein, N. et al. Targeted inhibition of galectin-1 gene expression in tumor cells results in heightened $\mathrm{T}$ cell-mediated rejection: a potential mechanism of tumorimmune privilege. Cancer Cel/ 5, 241-251 (2004).

95. Valenzuela, H.F. et al. O-glycosylation regulates LNCaP prostate cancer cell susceptibility to apoptosis induced by Galectin-1. Cancer Res. 67, 6155-6162 (2007).

96. Daniels, M.A., Hogquist, K.A. \& Jameson, S.C. Sweet ' $n$ ' sour: the impact of differential glycosylation on T cell responses. Nat. Immunol. 3, 903-910 (2002).

97. Comelli, E.M. et al. Activation of murine $\mathrm{CD}^{+}$and $\mathrm{CD} 8^{+} \mathrm{T}$ lymphocytes leads to dramatic remodeling of $\mathrm{N}$-linked glycans. J. Immunol. 177, 2431-2440 (2006).

98. Morgan, R. et al. N-Acetylglucosaminyltransferase V (Mgat5)-mediated N-glycosylation negatively regulates Th1 cytokine production by T cells. J. Immunol. 173, 7200-7208 (2004).

99. Bax, M. et al. Dendritic cell maturation results in pronounced changes in glycan expression affecting recognition by Siglecs and galectins. J. Immunol. 179, 8216-8224 (2007).

100. Lau, K.S. et al. Complex N-glycan number and degree of branching cooperate to regulate cell proliferation and differentiation. Cell 129, 123-134 (2007).

101. Grigorian, A. et al. Control of T cell-mediated autoimmunity by metabolite flux to $\mathrm{N}-$ glycan biosynthesis. J. Biol. Chem. 282, 20027-20035 (2007).

102. Togayachi, A. et al. Polylactosamine on glycoproteins influences basal levels of lymphocyte and macrophage activation. Proc. Natl. Acad. Sci. USA 104, 15829-15834 (2007).

103. Green, R.S. et al. Mammalian N-glycan branching protects against innate immune self-recognition and inflammation in autoimmune disease pathogenesis. Immunity 27, 308-320 (2007)

104. Van Dyken, S.J., Green, R.S. \& Marth, J.D. Structural and mechanistic features of protein $\mathrm{O}$ glycosylation linked to CD8 ${ }^{+}$T-cell apoptosis. Mol. Cell. Biol. 27, 1096-1111 (2007).

105. Kaneko, Y., Nimmerjahn, F. \& Ravetch, J.V. Anti-inflammatory activity of immunoglobulin G resulting from Fc sialylation. Science 313, 670-673 (2006).

106. Arnold, J.N. et al. The impact of glycosylation on the biological function and structure of human immunoglobulins. Annu. Rev. Immunol. 25, 21-50 (2007).

107. Ju, T. \& Cummings, R.D. Protein glycosylation: chaperone mutation in Tn syndrome. Nature 437, 1252 (2005).

108. An, G. et al. Increased susceptibility to colitis and colorectal tumors in mice lacking core 3-derived O-glycans. J. Exp. Med. 204, 1417-1429 (2007).

109. Feizi, T. \& Chai, W. Oligosaccharide microarrays to decipher the glycocode. Nat. Rev. Mol. Cell Biol. 5, 582-588 (2004). 